\title{
APLICACIÓN DE LAS POLÍTICAS DE LA UNIÓN EUROPEA EN MELILLA ENTRE 1986Y 2008: VALORACIÓN DE SUS EFECTOS Y PROSPECTIVA SOBRE MEJORAS (I)
}

\author{
JESÚS A. GARCÍA AYALA \\ Doctor en Ciencia Política y de la Administración por la Universidad de Granada \\ Licenciado en Ciencias Económicas y Empresariales por la \\ Universidad Complutense de Madrid \\ Director General de Fondos Europeos de la Ciudad Autónoma de Melilla
}

Resumen: En 1985, se pretendía que la integración de Melilla en la Unión Europea produjera un beneficio local neto, mediante un régimen especial que privilegiara el comercio y, como contrapartida, asumiera desventajas para otras actividades económicas. En 2009, existen indicios de que dicho régimen podría haber devenido en manifiestamente mejorable. En consecuencia, surge la necesidad de una valoración empírica y, de confirmarse los indicios, de una prospectiva sobre mejoras. Tras su investigación científica, se detalla la metodología observada, se sintetiza el núcleo de aquella y se anticipan sintéticamente las medidas susceptibles de adopción identificadas y las conclusiones alcanzadas.

Palabras clave: Melilla, Régimen especial, Régimen territorial, Régimen territorial especial, Prospectiva comunitaria, Política territorial, Política de cohesión.

Clasificación JEL: A12, A13, F13, F36, F51, F52, H24, H25, H27, H56, H71, H72, H73, H87, I31, N47, N77, O18, R11, R12.

Abstract: In 1985, it was sought that the integration of Melilla in the European Union produced a net local benefit, by means of a special regime that privileged the trade and, as compensation, it assumed disadvantages for other economic activities. In 2009, they exist indications that this regime could have become in professedly object of improvement. In consequence, the necessity of an empiric valuation arises and, of being confirmed the indications, of a prospective one about improvements. After their scientific investigation, the observed methodology is detailed, the nucleus of that is synthesized and, synthetically, they are early the identified susceptible of adoption measures and the reached conclusions.

Key words: Melilla, Special Regime, Territorial Regime, SpecialTerritorial Regime, Community Prospective, Territorial Policy, Cohesion Policy. 


\section{INTRODUCCIÓN}

Parte de los resultados de la investigación realizada para valorar empíricamente la evolución de los efectos de las políticas comunitarias desarrolladas en Melilla desde 1986, así como para disponer de un análisis de prospectiva sobre las posibilidades de mejora del vigente régimen especial de integración de Melilla en la UE, pasan a exponerse a continuación. El resto de los resultados se prevé incluirlos en una próxima publicación: Aplicación de las políticas de la Unión Europea en Melilla entre 1986 y 2008: valoración de sus efectos y prospectiva sobre mejoras (II), ${ }^{1}$ que se difiere hasta tanto sea defendida la tesis doctoral en la que se inscriben.

\section{CUESTIONES PREVIAS A LA INVESTIGACIÓN, HIPÓTESIS Y HERRAMIENTAS METODOLÓGICAS}

A continuación se detallan las principales cuestiones previas a la investigación, las hipótesis de partida y las herramientas metodológicas.

\section{PRINCIPALES CUESTIONES PREVIAS A LA INVESTIGACIÓN}

A nuestro juicio deben ser las siguientes: Elección; Planteamiento, objetivos y justificación; Marco teórico referencial y Alcance.

\subsection{Elección de la investigación}

Los tres aspectos principales a diferenciar en la misma deben ser: la realidad problemática resultante de precisar una situación, un espacio y un tiempo de referencia; la elección de factores instrumentales y los problemas a investigar dentro de estos.

\subsubsection{Realidad problemática}

La realidad a considerar para la elección de la investigación debería radicar en determinadas observaciones e investigaciones empíricas, que alertan sobre el hecho de

\footnotetext{
${ }^{1}$ En lo sucesivo "Informe II".
} 
que el modelo especial de integración de Melilla en la UE, vigente desde el 1 de enero de 1986, podría haber agotado su aportación positiva para dicha ciudad, e incluso haberse convertido en un obstáculo para su desarrollo económico y social en el siglo XXI. Si tuviéramos que citar al menos una de dichas observaciones y experiencias empíricas, a nuestro juicio deberíamos resaltar el hecho de que los indicadores del paro en Melilla vienen siendo de tal gravedad que indican que algo no va bien en la socio economía de la ciudad.

En definitiva, cabría acoger la alerta ${ }^{2}$ de que las contrapartidas comunitarias que se aceptaron desde 1986 en detrimento de las actividades económicas diferentes del comercio, en tanto sector primado, pueden estar resultando contraproducentes si la correlativa sobreprotección inicial de éste estuviera hoy vacía de contenido.

\subsubsection{Elección de factores instrumentales}

Entre los factores genéricos a considerar desde 1986, puede hacerse alusión a las siguientes transformaciones experimentadas desde entonces: el desarme arancelario impulsado desde la Organización Mundial del Comercio; las reducciones arancelarias acordadas por las Comunidades Europeas tanto dentro del régimen de preferencias generalizadas, como a través de regímenes preferenciales especiales y, en particular, mediante el otorgado a Marruecos; y la formalización entre las Comunidades Europeas y dicho país de, primero, el Acuerdo Euro mediterráneo (con desarme arancelario mutuo incluido) y, segundo, el Estatuto Avanzado otorgado por la UE a Marruecos recientemente.

Descendiendo al ámbito de los diferentes factores específicos, los que entendemos esenciales son dos: en primer lugar, la evolución y estado de situación de los efectos económicos y sociales originados por el régimen especial de integración de Melilla en la UE; en segundo lugar, la posible configuración contextual del potencial de mejora de dicho régimen.

\subsubsection{Selección de problemas a investigar}

Con respecto al primero de los citados factores específicos, el problema de investigación debería ser el siguiente: ¿Qué resultados aporta el análisis del régimen especial

\footnotetext{
disponible.

${ }^{2}$ Aportada tanto por las observaciones indiciarias aludidas como por la evidencia empírica
} 
de la UE vigente en Melilla desde 1986 y cuál es, dentro de dicho análisis, la valoración de efectos desde la evidencia empírica disponible?

En lo que se refiere al segundo factor, el problema de investigación seleccionado radicaría en la siguiente pregunta: ¿Cuáles son las posibilidades de mejora del régimen de integración de Melilla en la UE, incluido el refuerzo de la seguridad económica y social, mediante el impulso de acciones institucionales y políticas comunitarias?

\subsection{Planteamiento, objetivos y justificación}

Procedamos seguidamente a dar respuesta a las preguntas siguientes: ¿Cómo debería plantearse el problema objeto de investigación?, ¿Cuáles serían los objetivos de ésta? y ¿Qué razones la justificarían?

\subsubsection{Planteamiento del problema}

En el plano académico formal, la investigación debería plantearse con la concurrencia de las características esenciales para considerarse científica: versar sobre problemas reales; tratar tanto de contribuir al mejor conocimiento de éstos, como de aportar nuevas consideraciones y revisar las anteriores; y aportar elementos para su verificación por otros investigadores.

Además de tales características horizontales, debería tenerse en consideración otra asimismo concurrente, de carácter singular y estrechamente vinculada a la realidad. Se trata de que Melilla, junto con Ceuta, son dos territorios con una ubicación geoestratégica singular que repercute en su ámbito económico y en el social, así como en el de la seguridad común a ambos. Se trata de ciudades sujetas a desventajas específicas que, al igual que las causas de donde proceden, oscilan entre la permanencia y la persistencia. En tal contexto diferencial, debe valorarse el hecho de los escasos estudios e investigaciones existentes sobre dichas ciudades en comparación con los que se ocupan del resto del territorio nacional.

\subsubsection{Objetivos de la investigación}

En cuanto a los objetivos sobre el pasado y el presente, el principal debería ser el conocimiento acerca de si los resultados económicos y sociales imputables desde $1986 \mathrm{al}$ régimen especial de integración de Melilla en la UE, son positivos o negativos para dicha ciudad, y en qué medida lo son. En un plano subordinado, existirían objetivos de carácter 
instrumental que se ocuparían del balance del comercio de Melilla con el resto del mundo, de los flujos financieros con el resto de la UE, y de los resultados en convergencia real con las medias de España y la UE.

En lo que respecta a los objetivos sobre el futuro, el principal debería ser el conocimiento de la posible evolución de la situación vigente si no se adopta medida alguna y, alternativa o adicionalmente, el conocimiento de las posibilidades de mejora del régimen vigente Melilla-UE. En cuanto a los objetivos instrumentales, deberían, por una parte, conocer la posible evolución, en ausencia de intervenciones, de los efectos en materia de seguridad económica y social del régimen Melilla-UE vigente; y por otro lado, deberían procurar el conocimiento de las posibilidades para la elaboración de una nueva propuesta que incluyera la seguridad económica y social de dicha ciudad. ${ }^{3}$

\subsubsection{Justificación y viabilidad de la investigación}

La investigación debería ser elegida sobre la base de la concurrencia de determinadas justificaciones objetivas y subjetivas:

- En cuanto a razones de carácter objetivo, una aproximación debería ser la de las probables consecuencias teóricas; se estima que serían las siguientes: a) Aportación de una evaluación científica de las interrelaciones Melilla-UE entre 1986 y 2008, en términos de seguridad económica y social; b) Como efectos secundarios, podría quizá demostrarse si es posible el cambio hacia una alternativa diferente favorable para Melilla. Otra aproximación sería la de las posibles aplicaciones prácticas de la investigación, que se estima consistirían principalmente en que, llegado el caso, se facilitaría el estudiar y ofrecer alternativas concretas de cambio válidas como solución al problema de fondo.

- En lo que respecta a razones de carácter subjetivo, deberían cumplirse las cuatro reglas clásicas que hacen referencia a la conexión investigación-investigador: correspondencia entre los intereses mutuos, accesibilidad física a las fuentes necesarias, capacidad cultural para el manejo de las mismas, y experiencia con respecto al cuadro metodológico seleccionado.

${ }^{3}$ Como aspecto común a los objetivos señalados, cabe recordar que existen también otros de carácter intermedio, previos a los instrumentales acabados de citar (conocimientos específicos sobre fiscalidad y ayudas de Estado, sobre cuestiones clave en materia de seguridad, etc.). En el extremo opuesto, existe otra perspectiva a considerar, que permite hablar de objetivos de carácter global; éstos, al configurarse por agregación de los ya señalados, consisten en una serie consecutiva de metas, a activar cada una de ellas siempre que se vaya cumpliendo la anterior de manera positiva para la continuidad de la investigación. 
En coherencia con las razones anteriores, se conseguiría una estrecha vinculación de la investigación con la realidad, es decir, con la ciudad de Melilla, de manera que podría hablarse con propiedad de la existencia de una auténtica investigación aplicada.

\subsection{Marco teórico referencial}

Dentro de este marco global deben diferenciarse tres marcos específicos: el de referencia, el teórico en sentido estricto, y el conceptual.

\subsubsection{Marco de referencia}

En cuanto a los antecedentes de investigaciones a tener en cuenta, para su selección sería suficiente, dada la escasez de aportaciones conocidas, que guardaran una relación razonablemente significativa con la nueva investigación.

En lo que se refiere a los enfoques metodológicos a observar, habría que comenzar por explorar la utilización en el pasado de diversas teorías aplicables a la nueva investigación; y por otro lado, la posible existencia de una sola teoría completamente desarrollada, que fuera aplicable a la totalidad de la nueva investigación.

En otro orden de cosas, los resultados obtenidos de las investigaciones previas deberían ser recuperados y evaluados; por su parte, los retos planteados por los anteriores investigadores también deberían tenerse en cuenta; y en lo que respecta a los principales problemas pendientes, deberían integrase plenamente en la nueva línea de investigación; a nuestro juicio, los principales problemas pendientes de solución son dos: uno, el que aún no se han abordado, o lo han sido limitadamente, las repercusiones que una eventual incorporación de Melilla a la Unión Aduanera puede comportar en cuanto a la evolución de las ayudas de Estado y, en particular, de la fiscalidad; otro es la falta de investigaciones sobre la posibilidad de reconducir el perjuicio que, en el caso de la incorporación de Melilla a la Unión Aduanera, podrían sufrir determinados importadores melillenses.

\subsubsection{Marco teórico propiamente dicho}

Dentro de este marco deben incluirse dos cuestiones: la revisión de la bibliografía, y lo relativo a la construcción de la perspectiva teórica.

- En cuanto a la revisión de la bibliografía y otros materiales documentales, las principales dificultades que cabe señalar son las limitaciones y carencias de buen número de registros estadísticos 
oficiales; no obstante, hay que aclarar que tales dificultades pueden ser superadas mediante diversos instrumentos alternativos (evidentemente con más tiempo y esfuerzo).

- Con respecto a la adopción de una teoría o el desarrollo de una perspectiva teórica, deben diferenciarse dos situaciones, con sus tratamientos respectivos: la relativa al pasado y presente, en la que podría utilizarse una determinada perspectiva teórica que cuenta con abundancia de evidencia empírica (sin perjuicio de su previa revisión y actualización); la otra situación es la relativa al futuro, para la que, de continuar sin detectarse teoría aplicable algún, sería necesaria la construcción de una perspectiva teórica ad hoc.

\subsubsection{Marco conceptual}

Las definiciones de términos y conceptos clave deberían afectar principalmente a los términos "Seguridad", "Economía" y "Social”, a la confluencia de los conceptos de "Economía", "Social” y "Libertad”, y a los conceptos de "Seguridad económica", "Seguridad social" y "Seguridad económica y social".

En cuanto a las bases legales a utilizar, deberían estar constituidas por normas de diversas procedencias y naturalezas. Por materias, de carácter aduanero, económico, financiero, fiscal, laboral, presupuestario y sobre seguridad. Por el ámbito competencial del que surgen, Unión Europea (derechos primario y derivado), Reino de España (ámbitos legal y reglamentario) y Ciudad Autónoma de Melilla (ámbito reglamentario).

\subsection{Alcance de la investigación}

La investigación debería comprender un proceso que integrara sucesiva y coherentemente los cuatro componentes tipológicos de las mismas: exploratorios, descriptivos, correlacionales y explicativos; además, dicha integración no debería hacerse de forma fragmentada sino mediante un proceso continuo.

En consecuencia, el tipo de investigaciones y estudios a desarrollar debería ser función de las diferentes configuraciones temporales de las etapas a diferenciar; a saber: a) El pasado lejano, o búsqueda de lo acaecido hasta 1986, cuya investigación debería enmarcarse en la tipología histórica; b) El pasado próximo, o búsqueda de las claves de la evolución entre el pasado lejano y el pasado reciente (entre 1986 y 2008), cuya investigación debería enmarcarse en la tipología descriptiva; c) El presente, o búsqueda de lo que está sucediendo ahora (2008/2009), cuya investigación debería enmarcarse en la tipología correlacional; d) El futuro, o descripción de lo que sucederá en 2010 y años sucesivos, cuya investigación debería enmarcarse en la tipología explicativa y que constituye el núcleo esencial de la investigación. 
Entre los diversos autores cuyos criterios deberían tenerse en cuenta a efectos de las tipologías citadas y de, en particular, sus respectivas asociaciones a los diferentes entornos temporales a considerar, cabe citar a Baptista Lucio (2000), Fernández Collado (2000) y Hernández Sampieri (2000).

\section{HIPÓTESIS DE PARTIDA}

A partir de la asunción de las consideraciones acabadas de expresar, formularíamos las tres hipótesis siguientes:

$1^{\circ}$. Los efectos negativos sobre la seguridad económica y social de Melilla, imputables al régimen especial de integración comunitaria de dicha ciudad, y soportables desde 1986 como contrapartida ineludible a los efectos positivos de mayor importancia pretendidos por dicho régimen, hace ya algún tiempo que, haciendo abstracción de los de naturaleza fiscal y arancelaria, sobrepasan a estos efectos positivos.

$2^{\circ}$. La evolución futura de la situación actual, en cuanto a los efectos sobre la seguridad económica y social de Melilla imputables a su vigente régimen especial de integración comunitaria, en ausencia de modificación, supresión o novación de dicho régimen no irá a mejor e incluso tenderá a debilitarse y empeorar ostensiblemente.

$3^{\circ}$. A iniciativa de las instituciones competentes españolas, y propuesta y aprobación de las comunitarias, existen posibilidades de mejora significativa de los efectos sobre la seguridad económica y social de Melilla, alcanzables a través de la modificación o novación del régimen especial de integración comunitaria de dicha ciudad, incluida una eventual incorporación a la Unión Aduanera. Así, pueden incorporarse especialidades favorables, en las que, por una parte, se incluya la preservación y no se descarte la mejora de las ventajas actuales en materia de fiscalidad en general; y por otro lado, se incluya, para el caso de los importadores inicialmente perjudicados en costes arancelarios y fiscales, un tratamiento gradual, de regresividad atemperada, que impida efectos traumáticos sobre su seguridad económica y social.

\section{HERRAMIENTAS METODOLÓGICAS}

Las que se consideran necesarias deben diferenciarse en función del marco temporal al que se refieren: al pasado y el presente; o al futuro. 


\subsection{Herramientas sobre el pasado y el presente}

Los estudios previos en que debería basarse parcialmente la investigación a desarrollar responderían en concreto, como acabamos de indicar, a diversos tipos, en cada uno de los cuales deberían seguirse las metodologías diferenciadas ya relacionadas y suficientemente conocidas.

\subsection{Herramientas sobre el futuro}

A nuestro juicio resultarían concernidos los siguientes elementos: 1) Tipologías, conceptos y aspectos esenciales; 2) Técnica de escenarios y diseño de futuros; 3) Prospectiva estratégica y pautas observadas.

\subsubsection{Tipologías, conceptos y aspectos esenciales}

Una metodología apropiada para la investigación a desarrollar sería la denominada "Metodología prospectiva" y, dentro de ésta, la modalidad de "Prospectiva estratégica". En su instrumentación sería necesario conciliar en lo posible las posiciones a veces no coincidentes de los principales autores: Benavides Ilizaliturri (2004), Hevia Araujo (2001), Merello (1973), François (1977), Miklos (1998), Tello (1998), Barbieri (1992) y Godet (2000). ${ }^{4}$

\subsubsection{Técnica de escenarios y diseño de futuros}

Dentro de las técnicas para el diseño de ámbitos futuros -en el marco de la metodología prospectiva- la que se considera más apropiada para la investigación es una de carácter cualitativo; en concreto, la Técnica de Escenarios. Desarrollada principalmente por Godet (2000) y Maza Zavala (1990), se presenta como una síntesis de los conceptos de prospectiva y estrategia, ${ }^{5}$ y acoge en su seno la "Metodología de Escenarios" y los "Modelos de Escenarios". ${ }^{6}$

4 También se ha tenido en cuenta la convergencia, en estos últimos años, de la mayoría de las opiniones, criterios y doctrinas hacia un concepto de organización interna que posibilita el ajuste hacia lo nuevo y su creación.

${ }^{5}$ Su objetivo es la integración del análisis individual de tendencias, posibles sucesos y situaciones deseables, dentro de una visión global del futuro.

${ }^{6}$ Tratados ambos profusamente por Van der Heijden (1998). 


\subsubsection{Prospectiva estratégica y pautas observadas}

En lo relativo a ámbitos temporales, debería distinguirse entre uno de carácter exploratorio, cuya finalidad sería la identificación de los retos de futuro; y otro de naturaleza normativa, que se dirigiría a la identificación y concreción de las opciones estratégicas posibles.

En lo que se refiere a actitudes frente al futuro, la investigación debería culminar proporcionando una base documentada y válida que permitiera una actitud pro-activa para la materialización de los cambios deseados.

Como cuestiones esenciales, las preguntas sobre las que debería intentarse descubrir las claves necesarias para, desde una perspectiva científica, poder darles respuesta en las conclusiones; serían las siguientes: a) ¿Qué puede ocurrirle a Melilla si no se adopta medida alguna sobre el modelo mediante el que se inserta en la UE desde 1986? b) ¿Qué pueden hacer las autoridades competentes de las instituciones españolas, nacionales y melillenses, para reaccionar ante las causas de los eventuales efectos negativos acompañados de una actitud interna pasiva? c) ¿Qué pueden hacer asimismo dichas autoridades para afrontar con éxito los cambios que se precisen? d) ¿Qué pueden hacer además tales autoridades para incluso modificar el futuro en el sentido deseado?

\section{INVESTIGACIÓN PROPIAMENTE DICHA Y PRINCIPALES MEDIDAS IDENTIFICADAS COMO SUSCEPTIBLES DE ADOPCIÓN}

El núcleo de la investigación que el autor del presente informe ha desarrollado siguiendo las pautas acabadas de detallar lo constituyen siete capítulos agrupados en tres partes.

\section{PRIMERA PARTE}

Está conformada por el capítulo 1. Su objeto primordial lo constituye la integración analítica de las cuestiones relativas a la seguridad económica y social, en tanto envolvente integral del resto de la investigación. El tratamiento otorgado al concepto de seguridad económica y social se ha preferido que fuera amplio y, por tanto, próximo a un carácter integral desde las perspectivas conceptual y temporal.

En coherencia con lo anterior, el análisis realizado no se ha quedado en un plano temporalmente estático limitado a la situación en la actualidad, sino que ha introducido la 
perspectiva histórica; ello nos ha permitido no sólo conocer las claves de la situación en el presente, sino también anticipar en lo posible las del futuro próximo y, así, posibilitar el diseño de estrategias para el fortalecimiento de la seguridad de Melilla.

Por otra parte, la integración del análisis de la seguridad económica y social de Melilla en este marco amplio y complejo, ha implicado tomar en consideración tipologías de seguridad insoslayables, que comprenden tanto la vertiente militar en sentido estricto relativa a los dos Estados directamente concernidos (España y Marruecos), ${ }^{7}$ como la vertiente de las debilidades, amenazas y riesgos de contenido preferentemente territorial. ${ }^{8}$

En lo que respecta a la seguridad económica y social en sentido estricto, los grupos conceptuales en los que se ha diferenciado y clasificado el análisis son los siguientes: a) Diferencias esenciales España-Melilla-Marruecos $;{ }^{9}$ b) Interdependencias y dependencias bilaterales España/Melilla-Marruecos; c) Estrategias de desarrollo regional Marruecos/ Nador, por una parte, y España/Melilla por otra. Asimismo se han integrado en el análisis determinados factores de riesgo, o amenazas, de carácter transversal a todas las tipologías reconocidas: a) Contencioso del Sahara Occidental; b) Pesca en caladeros marroquíes; c) Tráfico de drogas; d) Inmigración ilegal; e) Auge del islamismo radical.

En cuanto a los elementos instrumentales utilizados, han consistido esencialmente en diversos textos, datos y cifras de fiabilidad contrastada relacionados de manera amplia con la interrelación España/Melilla, por una parte, y Marruecos/Nador, por otra; entre la diversa documentación bibliográfica tenida en consideración destaca la utilización de Tratados y Convenios internacionales, documentos oficiales y de la sociedad civil de diversos países, e informes de distintos organismos internacionales.

\section{SEGUNDA PARTE}

Está conformada por los capítulos 2, 3 y 4. Su objeto de conocimiento lo ha constituido el estado de situación del vigente régimen especial de Melilla en la UE. En concreto se ha ocupado de la delimitación, estudio, análisis y, en su caso, interpretación del estado de situación de Melilla en aquellas cuestiones que, relacionadas con la investigación desarrollada, tienen a priori un interés relevante. Dicho interés se ha plasmado

${ }^{7}$ Comparación de capacidad bélica, de alianzas internacionales y otros factores, y de índices sobre paz y militarización.

${ }^{8}$ Superficie terrestre, aguas jurisdiccionales y espacio aéreo.

${ }^{9}$ Desarrollo humano, capacidades básicas y libertades económicas. 
en la necesidad de conocer los trabajos científicos más recientes que han aportado nuevos conocimientos al análisis del régimen especial Melilla-UE, conocimiento que ha permitido plantear alternativas para la optimización de efectos en el futuro.

En el capítulo 2 se analizan las características y configuración del régimen especial de integración de Melilla en la antigua CE, diferenciando entre las características asociadas a políticas comunitarias instrumentadas en Melilla de manera diferente o igual (total o parcialmente) al caso del régimen general de España. Además, se profundiza en el análisis del contenido (normas y su interpretación) y aplicación (prácticas y su documentación) de la parte del régimen especial de Melilla diferenciada del régimen general de España, especialmente en el caso de las políticas de mayor importancia para dicha ciudad y en el de las contribuciones presupuestarias de la misma a la UE.

En el capítulo 3 se analiza e interpreta el estado de situación de Melilla en cuanto a determinados efectos que, en principio, son susceptibles de estar causados en alguna medida por la aplicación de las políticas comunitarias inherentes a su régimen especial comunitario. Se trata por tanto de una "evaluación de referentes empíricos" y, en concreto, de los aportados por las más recientes investigaciones sobre materias conexas con el objeto de estudio de la tesis. En el desarrollo de este capítulo los distintos referentes científicos que son objeto de análisis se agrupan en tres apartados, a saber: uno sobre el balance del comercio entre Melilla y el resto del mundo, otro sobre el balance de flujos financieros entre Melilla y el resto de la UE, y el último sobre el proceso y estado de situación de la convergencia económica y social de Melilla con las medias de España y la UE. En lo relativo al análisis de resultados, hemos pretendido dar respuesta a los cuatro grandes tipos de análisis de datos a disposición del investigador y que, según criterio de los principales autores consultados (Balso, 2001 y Lewis, 2001) se caracterizan por perseguir, bien una mayor descomposición de los datos, bien una búsqueda de relaciones entre las variables medidas que faciliten las necesarias matizaciones, bien el establecimiento de contrastes, bien la observación de las eventuales excepciones.

El capítulo 4 se ha dedicado al estudio de las aportaciones científicas seleccionadas. Éstas han sido escasas debido a dos factores: en primer lugar, en su selección ha primado que Melilla (o Ceuta) fuera su objeto de estudio, o al menos una parte del mismo tratada con el suficiente detalle; y en segundo término, se ha velado porque se contuvieran, expresa o implícitamente, resultados o conclusiones en términos de retos identificados y propuestas planteadas para la mejora de la situación integral de Melilla (o de Ceuta). Para el tratamiento de resultados y el análisis e interpretación subsiguientes, se ha dispuesto exactamente de seis trabajos científicos. 


\section{TERCERA PARTE}

Está conformada por los capítulos 5, 6 y 7. Su objeto de conocimiento se dirige a las posibilidades de mejora integral del vigente régimen especial Melilla-UE, enfocándolas además hacia el posible impulso al efecto desde el ámbito comunitario. Este impulso se refiere tanto al área de las acciones de carácter institucional en sentido estricto, como al área de aplicación de las diferentes políticas. Es de resaltar además, que dichas posibilidades de mejora engloban los aspectos de seguridad definidos en la tesis. En cuanto a la prospectiva estratégica utilizada, ha sido enfocada a las posibilidades concretas en las áreas cuyo interés para Melilla se ha demostrado esencial; en coherencia con ello, se han abordado tanto las actuaciones adoptables por organismos y autoridades regionales y nacionales, ${ }^{10}$ como las actuaciones adoptables directamente desde el ámbito comunitario; ${ }^{11}$ Dentro de las actuaciones concernidas, cabe destacar la especial atención prestada a las necesarias formalidades procedimentales, en particular en materia de distribución y residencia de las correspondientes competencias. Las soluciones que finalmente se ofrecen se caracterizan porque, más allá de su idoneidad técnica para resolver problemas, integran el deseable componente de mejora y fortalecimiento de los elementos identificados como claves para la seguridad económica y social de Melilla.

El capítulo 5 se dedica a la prospectiva, tanto enfocada a la preservación y mejora del tratamiento a Melilla en materia de fiscalidad, como hacia especialidades para la eventual integración de dicha ciudad en elTerritorio Aduanero Común. La sistemática que se sigue en cuanto a la política fiscal diferencia las dos grandes áreas existentes: la fiscalidad directa y la indirecta: en el caso de la directa, dado que la competencia exclusiva sigue radicando en los Estados miembros, pero no obstante en determinados supuestos se produce la intervención de la propia UE, se exploran las posibilidades derivadas de la dinámica comunitaria en la aproximación de esta fiscalidad; en el caso de la indirecta, habida cuenta que la UE dispone de importantes competencias y muestra una clara vocación de expansión, se examinan las potencialidades dimanantes de la dinámica en la armonización de la misma; a su vez, dentro de esta última fiscalidad se tratan diferenciadamente el caso del Impuesto sobre el Valor Añadido y el de los Impuestos Especiales. En materia aduanera se abordan

\footnotetext{
${ }^{10}$ Por ejemplo tratamientos dentro de la normativa fiscal.

${ }^{11}$ Por ejemplo tratamientos enmarcados en la normativa aduanera o sobre ayudas de Estado, dentro de los cuales se incluyen a su vez instrumentos diversos, como, entre otros, el de la Zona Franca y el del Registro Especial de Buques
} 
las siguientes problemáticas: la relacionada con la aplicación del Código Aduanero Comunitario; las posibilidades derivadas de la configuración normativa de la figura de la Zona Franca, especialmente la de nueva generación; asimismo, las posibilidades que, para proporcionar cobertura normativa comunitaria a la creación de actos jurídicos especiales, ${ }^{12}$ podría aportar la aprobación de un Programa de Opciones Específicas por la lejanía y la insularidad de la ciudad de Melilla (POSEIMEL). Por su parte, el tratamiento a efectos de prospectiva ha sido similar en todos los casos: primero, síntesis del estado de situación referido a la normativa vigente, a su aplicación y a la interpretación que en su caso efectúa al respecto elTribunal de Justicia; ${ }^{13}$ segundo, perspectivas futuras desde un enfoque que adopta como referencia los principales actos comunitarios conexos a normas seleccionadas, en el contexto tanto del Tratado de Niza como del de Lisboa; y tercero, implicaciones susceptibles de afectar a Melilla en el contexto de una modificación futura de su régimen fiscal especial dentro de la UE. Todo ello debe entenderse en el contexto de una posible integración de Melilla en la Unión Aduanera, plena aunque con especialidades en el curso de un proceso transitorio, a modular para la aplicación gradual de las disposiciones propias del Código Aduanero Comunitario en general y del Arancel Aduanero Común en particular.

El capítulo 6 explora la realidad potencialmente aplicable a favor de Melilla constituida por las ayudas públicas en general y las de Estado en particular. Ambas presentan una casuística compleja, a analizar en función de su compatibilidad o no con el mercado común. En su desarrollo se ha procedido mediante la siguiente sistemática: en primer lugar, se ha abordado el estado de situación de la normativa comunitaria en materia de ayudas de Estado, incluidas las excepciones en general; en segundo término, se ha profundizado en las posibilidades de las excepciones en materia regional y de transportes, entre otras tipologías de interés; en un tercer plano, se ha abordado el estudio de la jurisprudencia más reciente con interés para Melilla, constituida esencialmente por cuatro sentencias clave delTribunal de Justicia; en cuarto lugar, se han explorado las perspectivas futuras de carácter objetivo sobre la evolución de las ayudas de Estado; y en quinta posición se ha concluido con las implicaciones que de todo lo anterior se siguen para el futuro del régimen especial de Melilla en la UE, a partir de la normativa y praxis comunitarias y de la labor interpretativa del Tribunal de Justicia. ${ }^{14}$

${ }^{12}$ Como entre otros posibles la aprobación de un régimen especial de abastecimiento.

${ }^{13} \mathrm{O}$, en su caso, el Tribunal de Primera Instancia.

${ }^{14}$ Así como del Tribunal de Primera Instancia. 
Finalmente, el capítulo 7 acoge la prospectiva sobre alternativas para mejorar la seguridad integral de Melilla, en el contexto de una incorporación a la Unión Aduanera que esté dotada de las modulaciones necesarias para contrarrestar los efectos estructurales de las desventajas permanentes de carácter natural y geográfico. Los elementos instrumentales utilizados han consistido preferentemente en los siguientes:'a) Posibles implicaciones a favor de Melilla de las directrices estratégicas comunitarias en materia de cohesión; b) Precedentes para Melilla del tratamiento modulado de la normativa comunitaria a determinados países y territorios asociados a la UE, o bien adheridos a la Unión Aduanera de forma especial; c) Profundización en el caso de los precedentes de modulaciones de la normativa comunitaria a favor de las Regiones Ultraperiféricas enumeradas expresamente en el artículo 299.2 TCCE; d) Análisis de la tipología de las regiones que, sin estar enumeradas en el citado precepto, podrían, según diversos autores (Asín Cabrera, 1988 y Fornieles Gil, 2005-2006) beneficiarse del mismo por reunir las condiciones exigibles; e) Concreción de alternativas para la modulación de la legislación, y para la correspondiente aplicación de las políticas comunitarias, a favor de los territorios considerados incluidos implícitamente en el reiterado artículo 299.2 TCCE;f) Síntesis de las medidas y procedimientos susceptibles de aplicación en el marco de decisiones estratégicas a adoptar políticamente para la obtención por Melilla de un mejor tratamiento comunitario. Este capítulo incluye finalmente un elenco de medidas susceptibles de adoptarse a favor de Melilla por parte de las autoridades competentes, esencialmente en el ámbito comunitario, mediante decisiones que, en principio y en tanto no se racionalicen científicamente, se inscribirían en el ámbito político; la tipología y número de medidas de cada una de las cinco tablas ofrecidas son las siguientes: 1) Modulaciones en la política de cohesión económica, social y territorial (30 medidas); 2) Especialidades en las demás políticas horizontales (79 medidas); 3) Especialidades en las políticas sectoriales (43 medidas); 4) Especificidades en las políticas relativas a la configuración, estructura, organización y funcionamiento del marco institucional de la UE (16 medidas); y 5) Especificidades en las políticas españolas permitidas por el acervo comunitario (5 medidas). ${ }^{15}$

\footnotetext{
${ }^{15}$ El detalle de todas estas medidas se reserva para el Informe II.
} 


\section{CONCLUSIONES DE LA INVESTIGACIÓN DESARROLLADA}

Las conclusiones obtenidas de la investigación desarrollada se inscriben en tres grupos conceptuales a diferenciar. En consecuencia, se formulan diez conclusiones diferenciadas en tres grupos: a) Las relativas a cada una de las hipótesis formuladas; b) Las referidas a cada una de las preguntas planteadas para la investigación; c) Una conclusión final. A continuación se procede a una somera síntesis de las principales. ${ }^{16}$

\section{PRIMERA CONCLUSIÓN}

La comprobación de la primera hipótesis formulada demuestra que ésta resulta apoyada en su totalidad de acuerdo a la ponderación de los resultados obtenidos en la investigación realizada. Las concreciones de esta conclusión diferencian y detallan los siguientes ámbitos: a) Políticas de competencia y de libre circulación de personas, servicios y capitales, política de cohesión y saldo presupuestario Melilla-UE; b) Aplicación de la política de fiscalidad; c) Aplicación de la política aduanera.

\section{SEGUNDA CONCLUSIÓN}

La comprobación de la segunda hipótesis formulada demuestra asimismo que ésta resulta apoyada en su totalidad de acuerdo a la ponderación de los resultados obtenidos en la investigación desarrollada. Las concreciones de esta conclusión diferencian y detallan los efectos sobre la seguridad económica y social Melilla de: a) Determinadas políticas comunitarias distintas a la fiscal y a la aduanera; b) Política de fiscalidad; c) Política reguladora de la actividad aduanera; d) Confluencia de políticas (efectos ponderados).

\section{TERCERA CONCLUSIÓN}

La comprobación de la tercera hipótesis formulada demuestra unos resultados a diferenciar. En efecto, de acuerdo a los datos obtenidos en la presente investigación, tanto la posibilidad de mejora de las actuales ventajas fiscales dentro de una modificación o novación del vigente régimen especial Melilla-UE, como la posibilidad de preservación

\footnotetext{
${ }^{16}$ Ibídem.
} 
de las actuales ventajas fiscales dentro de una novación del vigente régimen especial MelillaUE resultan apoyadas en un determinado supuesto marco y no apoyadas en otro. Aparte de los detalles sobre ambos supuestos, ${ }^{17}$ otras concreciones a diferenciar y detallar en el caso de esta conclusión son las siguientes: a) Mejora que determinadas modificaciones o novaciones del actual régimen especial Melilla-UE pueden comportar sobre la seguridad económica y social de dicha ciudad; b) Posibilidad de que el actual régimen especial de Melilla dentro de la UE sea objeto de una modificación o novación que incluya la preservación y no descarte la mejora de las ventajas actuales en materia de fiscalidad; c) Posibilidad de que el actual régimen especial de Melilla dentro de la UE sea objeto de una modificación o novación que incluya un tratamiento gradual (de regresividad atemperada y temporalidad dilatada) que evite posibles efectos traumáticos en el orden económico y social a los importadores sujetos, en principio, al mayor coste arancelario y fiscal que conlleva el operar dentro de la Unión Aduanera; d) Posibilidad de que el actual régimen especial de Melilla dentro de la UE sea objeto de una modificación o novación que incluya otras especialidades favorables (horizontales y regionales preferentemente); e) Posibilidad de que la modificación o novación del régimen especial de integración comunitaria de Melilla, pueda ser llevada a término a iniciativa de las instituciones competentes españolas, y propuesta y aprobación de las instituciones competentes comunitarias.

\section{CONCLUSIONES CUARTA A NOVENA}

Suponen un desarrollo de las conclusiones acabadas de establecer. ${ }^{18}$

${ }^{17}$ Las cuales, junto con aquellas a que se hace referencia seguidamente, se incluirán en el Informe II.

${ }^{18}$ Se incluirán en el Informe II. Puede avanzarse no obstante que las preguntas a las cuales, planteadas dentro de la investigación desarrollada, se asocian estas conclusiones son las que se indican seguidamente. Preguntas asociadas a la conclusión número 4: ¿Cuáles son las claves del ámbito de la seguridad a tener presentes en la mejora de la realidad económica y social de Melilla? y, en dicho contexto, ¿Existen posibilidades reales de fortalecimiento de la seguridad económica y social de Melilla mediante las instituciones y políticas comunitarias? Pregunta asociada a la conclusión número 5: ¿Cuáles son hoy día los aspectos esenciales del sistema económico y fiscal de Melilla vigente desde 1986, distinguiendo entre su parte diferenciada totalmente del régimen general para la Península y Baleares y su parte diferenciada parcialmente? Pregunta asociada a la conclusión número 6: ¿Qué resulta de la evaluación de referentes empíricos acerca de los balances para Melilla de su comercio con el resto del mundo, de los flujos 'financieros con el resto de la UE y de la convergencia económica 


\section{DÉCIMA CONCLUSIÓN}

Se trata de una conclusión final sobre lo que pueden hacer, acumulativamente, las autoridades competentes. Consideramos que a efectos de la presente exposición puede ser suficiente, en principio, limitarnos a la enunciación de las cuestiones a las que va respondiendo esta última conclusión; a saber: primero, lo que sí pueden hacer las autoridades competentes de las instituciones españolas, nacionales y melillenses, para reaccionar ante las causas de unos efectos negativos que, hasta ahora, han venido siendo acompañados de una actitud interna pasiva; segundo, lo que sí pueden hacer también dichas autoridades, para afrontar con éxito los cambios que se precisen; tercero, lo que sí pueden hacer además tales autoridades, para incluso modificar el futuro en el sentido deseado desde una perspectiva económica y social; cuarto y último, lo que asimismo sí pueden hacer las citadas autoridades para, adicionalmente, asegurar el futuro en el sentido deseado desde una perspectiva de seguridad internacional para Melilla.

\section{BIBLIOGRAFÍA}

ASÍN CABRERA, M.A. (1988): Islas y Archipiélagos en las Comunidades Europeas. Madrid:Tecnos, BALSO, M. y LEWIS, A. D. (2001): First Steps: A Guide to Social Research. Segunda edición. ITP, Nelson.

BARBIERI MASINI, E. (1992): Why Futures Studies? Londres: Grey Seal Books, BENAVIDES ILIZALITURRI, L. G. (2004): Metodología prospectiva. México - Veracruz:

Disponible en Web: <http://www.sabersinfin.com/index.php?option $=$ com_content\&task $=$ view\&id $=182 \&$ Itemid $=12>$

y social con el resto de España y de la UE? Pregunta asociada a la conclusión número 7: ¿Existen posibilidades reales de preservación y mejora del tratamiento a Melilla en materia de fiscalidad directa e indirecta, así como en materia de régimen aduanero, en un escenario de modificación o novación del actual régimen especial Melilla-UE que incluya una eventual integración en el Territorio Aduanero de la Comunidad? Pregunta asociada a la conclusión número 8: ¿Existen posibilidades reales de perfeccionar el tratamiento a Melilla en materia de ayudas de Estado? Pregunta asociada a la conclusión número 9: ¿Existen alternativas reales para que, en el caso de una eventual incorporación de Melilla a la Unión Aduanera, se obtenga una modulación que, en atención a los efectos estructurales de las desventajas permanentes y a su tratamiento histórico, permita mejorar la seguridad económica y social de dicha ciudad? 
BERGER, G. (1964): Phomenologie du temps et prospectiva. Primera edición. París: PUF. FORNIELES GIL, A. (2005-2006): La fiscalità delle regioni ultraperiferiche [Tesis doctoral]. Bolonia: Alma Mater Studiorum - Università di Bologna.

FRANÇOIS, Ch. (1977): Introducción a la prospectiva. Primera edición. Buenos Aires: Pleamar.

GARCIA AYALA, J. A. (2007): El régimen especial de Melilla en la Unión Europea y sus efectos: una valoración de la evidencia empírica disponible hasta 2006 (seguridad versus inseguridad de Melilla en los ámbitos económico, social y territorial, como efecto de las decisiones adoptadas ad hoc en el contexto de la adhesión de España a las Comunidades Europeas: un estudio empírico desde 1986 hasta 2006). Informe inédito [Cursos de Doctorado en la Universidad de Granada. Período de investigación], 549 p., 21 cuadros, 4 documentos, 8 esquemas, 3 figuras, 96 gráficos, 128 tablas.

(2006): Planteamiento de la problemática de seguridad y defensa en la región mediterránea: Estado de situación en 2006. Informe inédito [Cursos de Doctorado en la Universidad de Granada. Período de docencia], 64 p.

GODET, M. et al. (2000): La caja de herramientas de la Prospectiva estratégica. Cuarta edición actualizada. París: Prospektiker - Laboratoire d'Investigation Prospective et Stratégique.

(2007): Prospectiva Estratégica: problemas y métodos. Segunda edición. París: Prospektiker - Laboratoire d'Investigation Prospective et Stratégique.

HERNANDEZ SAMPIERI, R.; FERNANDEZ COLLADO, C.; BAPTISTA LUCIO, P. (2000): Metodología de la Investigación. México: McGraw Hill.

HEVIA ARAUJO, O. R. (2001): Metodología de escenarios: ¿Utopía o Concreción prospectiva en las Ciencias Sociales? [s.l.]: ca. Disponible en Web: <http://www.iaeal.usb.ve/90/ 90-3.pdf>

MAZA ZAVALA, D. F. (1990): "Los procesos económicos y sus perspectivas", Nuevas estrategias de desarrollo económico ante la crisis actual de Venezuela y Latinoamérica, 299 p. Caracas: Biblioteca de la Academia Nacional de la Historia.

MEREllO, A. (1973): Prospectiva, Teoría y Práctica. Primera edición. Buenos Aires: Guadalupe.

MIKLOS, T. y TELLO, M. E. (1998): Planeación Prospectiva: Una Estrategia para el diseño del futuro. Primera edición. México: Limusa.

VAN DER HEIJDEN, K. (1998): Escenarios. El arte de prevenir el futuro. Primera edición. México: Panorama. 